\title{
THE PROGNOSIS OF NEPHRITIS IN CHILDHOOD
}

\author{
BY \\ NORMAN S. CLARK \\ From the Department of Child Health, University of Aberdeen
}

(RECEIVED FOR PUBLICATION FEBRUARY 6, 1956)

It has long been recognized that comparatively few cases of nephritis in childhood die in the early stages of the disease and it is widely believed that the great majority of cases recover completely. Hebert (1952) has summarized the results of many of the long-term studies of nephritis in childhood published in the British and American literature in the past 30 years. In the majority of these reports complete recovery was claimed in from $70 \%$ to $97 \%$ of the survivors from the acute illness. Only four of the reports quoted were published in this country and of these only one (Giles, 1947) has appeared in the last 20 years. I have found three additional papers on this subject in the British literature in that period (Cass, 1939; Tallerman and Burkinshaw, 1939; Payne and Illingworth, 1940). Tallerman and Burkinshaw (1939) and Giles (1947) support the majority view that over $70 \%$ of cases recover completely. Cass (1939) found $64 \%$ healed of 88 cases with an acute haemorrhagic onset. On the other hand, Payne and Illingworth (1940) found evidence of active or latent disease in 62 out of 89 cases seen from one to 12 years after the initial attack. Their findings support a minority view, strongly held in America, that the long-term prognosis in nephritis is much poorer than is popularly supposed, and with Snoke (1937) they maintain that a failure to recognize the latent stage has been responsible for undue optimism in many of the published reports claiming a high proportion of complete recoveries. Snoke, indeed, claimed that 'no patient who has had nephritis can be considered as healed until repeated quantitative studies of concentrated urine, i.e., Addis counts, have given the kidney a clean bill of health'. On this basis he considered that only 57 of 154 patients followed up had healed completely. Murphy and Rastetter (1938) found a very similar recovery rate in a study of 105 patients under 30 years of age. In a subsequent paper published from Rochester, however, Snoke (1939) reported a very different long-term prognosis; here, using the same standards of cure, he found a recovery rate of $72 \%$ in 106 patients as compared with $37 \%$ in his original series reported from Stanford University Medical School. He suggested that sampling and a geographical difference in the manifestations of nephritis might explain this discrepancy.

Because of these differences of opinion and in particular of the extremely poor prognosis suggested by Payne and Illingworth (a view which, so far as I know, has been subsequently contradicted in this country only by Giles (1947)), it appears worth while to present the results of a follow-up study of 265 consecutive cases of nephritis admitted to the Royal Aberdeen Hospital for Sick Children between 1934 and 1952.

\section{Material}

The case material for the present study has been briefly described in a previous paper (Clark, 1956) in which it was shown that the cases did not appear to fall into two clearly defined types in accordance with the Ellis (1942) classification, and it was suggested that all the manifestations of nephritis were different expressions of one disease process. The 265 cases will, therefore, all be considered together when assessing the prognosis.

This study was undertaken in the first instance in the course of a clinical trial of an antihistamine drug (mepyramine maleate) in the treatment of nephritis. Initial experience with this drug suggested that it might be of value (Craig, Clark and Chalmers, 1949) and the last 62 consecutive cases of the series reported here were treated with this drug for varying periods. No evidence was obtained that mepyramine maleate influenced the mortality or the duration or severity of the initial illness, nor did it diminish the incidence of cases left with signs of permanent renal damage. This confirms the views of Thomson (1949) and Lawson (1951) based on smaller series of cases followed for relatively short periods. The 62 cases so treated are therefore included with the previous 203 cases for the purpose of the present paper. 


\section{Mortality in the Initial Illness}

Twenty patients $(7.5 \%)$ died within one year of the onset of the disease, 16 of them within four weeks. This figure does not, however, represent the mortality to be expected at the present date; 13 of these deaths occurred in the first 100 cases, admitted between 1934 and 1939, and only three in the last 100, admitted between 1945 and 1952. This fall in mortality is largely due to the effects of sulphonamides and antibiotics in controlling antecedent or intercurrent infection. At least seven of the first 13 deaths can be attributed largely to infection (meningitis, septicaemia, pneumonia) with nephritis playing only a secondary role. Of the remaining six deaths, four occurred within three days of admission to hospital, three of them with evidence of circulatory failure. Necropsy in these cases confirmed the diagnosis of nephritis and showed no other cause of death. The three deaths in the last 100 cases were all attributable solely to nephritis.

\section{Long-Term Prognosis}

Of the 245 survivors from the initial illness 67 were followed up for less than one year after the onset of their illness and remained untraced at the final follow-up examination. The majority of these cases were considered to have recovered completely when they were last seen, but they will not be further considered here as the follow-up period was so short and the evidence of recovery in some cases incomplete. There remain 178 cases whose present status will now be discussed.

Three cases are known to have died at home, six, eight and 10 years after the onset of their illness, death in each case being certified as due to chronic nephritis. One of these ran a somewhat unusual course in that she had at least 15 distinct relapses with gross haematuria and oedema, many of them necessitating re-admission to hospital; during the remissions between these attacks she was at first relatively well and her blood pressure did not begin to rise until over three years after her first attack. When last seen shortly before her death she had generalized oedema, marked hypertension and gross haematuria but only slight azotaemia.

One hundred and fifty-six cases were examined at a final follow-up examination carried out from one to 13 years after the onset of the illness (in only 13 of them was the interval less than two years). The remaining 19 were unable to attend for examination but were reported to be in normal health. In four cases this statement was supported by the fact that the patient was now overseas, having been accepted in H.M. Forces in the highest physical category and in five others by a report from the family doctor. In 12 of these cases the attack of nephritis had occurred more than eight years previously.

At the final follow-up examination of the 156 cases a record was made of any illnesses suffered since the attack of nephritis, a general physical examination, including blood pressure estimation, was carried out and a single specimen of urine was examined for albumin and by microscopy of the centrifuged deposit. In the case of children admitted between 1935 and 1945 this examination was made in the course of a previous investigation (Russell, 1949); in the remaining children the examination was carried out at the ward follow-up clinic and, in virtually all of these, the results of the final examination were supported by one or more previous examinations carried out at intervals of a few months.

In 115 cases no abnormality was detected at the final examination. In 14 there was evidence of latent nephritis; all these patients had remained clinically well but 10 showed albumin + or -+ in the urine, two had a trace of albumin and scanty red cells, one had scanty red cells only and one a trace of albumin and a blood pressure of $120 / 90 \mathrm{~mm} . \mathrm{Hg}$ at the age of 8 years.

Eight cases showed evidence of active nephritis. In six of these there was moderate or heavy albuminuria and a history of recurrent episodes of oedema and, in the remaining two, moderate albuminuria and cylindruria and hypertension.

In 19 cases a trace of albumin was found in the urine but the examination was otherwise negative; these cases have been classified as 'uncertain' because of the doubtful significance of this finding in a single non-catheter specimen of urine.

These results are summarized in the following table and it is obvious that they coincide with the majority view on the prognosis of nephritis in childhood.

TABLE

FATE OF 178 CASES TRACED AFTER 1 TO 13 YEARS FROM THE ONSET OF NEPHRITIS

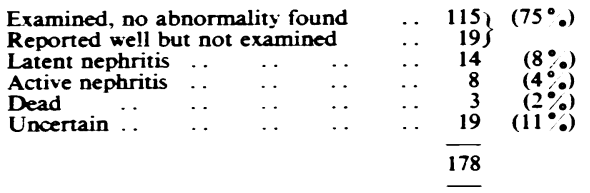

As Snoke (1937) and Payne and Illingworth (1940) maintain that the criteria of cure adopted in the majority of published reports are insufficiently strict to detect latent nephritis, it appeared desirable to investigate some of the cases further and 34 of the 
more recent cases were recalled for examination and the performance of Addis counts. (The Addis count technique is described and the range of normal figures defined in the Appendix.) The 34 cases were selected because they were not enuretic and because they lived near enough to attend the hospital without inconvenience and to deliver the Addis count specimens within a few hours of collection. They were unselected in respect of the severity and duration of their initial attack of nephritis and could fairly be considered a representative sample in that respect. They consisted of 24 patients considered healed at the previous examination, four patients considered to have latent nephritis and six who were classified as 'uncertain'.

Counts were performed on one occasion in 12 of the 24 healed cases and on two or more occasions in the remaining twelve. The red cell counts ranged from 0 to 408,000 , being lower than 100,000 in 30 specimens. Cast counts between 2,000 and 7,000 were found in four specimens only; no casts were seen in any other specimen. A trace of albumin was found in one specimen only (from a girl); a second specimen from the same child was negative. Addis counts have, therefore, failed to reveal any additional cases of latent disease among these 24 cases passed as healed on routine examination.

In the four cases considered to show evidence of latent nephritis Addis counts gave the following results:

\begin{tabular}{c|r|r|c}
\hline Case No. & Red Cells & Casts & Albumin \\
\hline A12 & 360,000 & 7,000 & Trace \\
A23 & $2,970,000$ & 0 & Trace \\
& 760,000 & 0 & Trace \\
A44 & 670,000 & 7,000 & + \\
& 610000 & 0 & Nil \\
A49 & 200,000 & 5,000 & Trace \\
& $1,820,000$ & 29,000 & + \\
& & 0 & Trace \\
\hline
\end{tabular}

Except in A12, therefore, Addis counts gave further support to the diagnosis of latent nephritis.

In four of the six cases classed as 'uncertain' Addis counts were normal and the specimens were free from albumin. It appears probable that the trace of albumin found on routine examination in these cases was not significant and that these four cases might also be considered healed.

Of the two remaining cases, A35 had an Addis count of 905,000 red cells and 6,000 casts and the specimen contained albumin + , while A24 had five normal counts during the course of one year (red cells ranged from 87,000 to 208,000 and casts from 0 to 7,000); each specimen contained a trace of albumin, however, and a catheter specimen taken at the time of the last count also showed a trace of albumin. Both cases should probably be classed as latent nephritis despite the succession of normal counts in A24.

\section{Discussion}

It is clear, therefore, that Addis counts have modified our views on the final status of these children only in the cases classified as 'uncertain' on routine examination. It appears also from the results quoted from Case A24 that the red cell and cast counts may be repeatedly normal despite persistent slight albuminuria. My impression of the Addis count (based on over 250 counts, personally performed, on these and other cases) is that it is a time-consuming technique with, inevitably, a fairly wide margin of error; it is of value in assessing the doubtful case in which the results of routine examination are equivocal and for certain specific purposes in research in which it may be desirable to have an objective measurement of haematuria; in the practical management of nephritis, however, the information which it gives does not justify the considerable expenditure of time which its routine employment would entail.

If, as seems possible, two-thirds of the 19 cases classified as uncertain are healed, they should at least counterbalance the possibility that some of the 19 cases reported well but not examined have in fact a latent nephritis. This further investigation, therefore, has given no grounds for revising the views summarized in the table on the ultimate outcome of the disease.

The difference between this result and the findings of Payne and Illingworth is difficult to explain. The follow-up period is the same in the two series. Payne and Illingworth recorded the blood pressure and examined the urine on two separate occasions at their follow-up examination; they estimated the amount of albumin in the urine quantitatively and did not perform Addis counts but regarded as pathological more than one red cell per one-sixth field in the centrifuged deposit. They also estimated the blood urea but found this raised in one case only. Their final examination was therefore somewhat more stringent than the final examination of the cases in the present series but not more so than in those 34 cases re-examined and submitted to Addis counts. It would have been reasonable to expect that at least a few additional cases of latent nephritis would be revealed by this further examination in view of their finding of 62 cases of latent disease among 89 cases examined. It is unlikely that selection of cases can account for this discrepancy. The present series includes all children admitted to hospital with nephritis from the North-Eastern Hospital Region of Scotland. The Hospital for 
Sick Children, Great Ormond Street, does not draw its cases from any defined area and has always tended to attract unusual or severe cases from a wide area; acute nephritis is, however, an unpredictable disease in which the severity of the early symptoms seems to have little bearing on the long-term prognosis and, while selective admission of severe cases might well result in high mortality figures for the initial illness, it is unlikely to increase to any marked degree the proportion of cases passing into the latent stage. It may well be that there is some foundation for the suggestion made by Snoke (1939) and by Payne and Illingworth that the manifestations of nephritis vary in different parts of the country.

Rebation of Initial Symptoms to End-results. An attempt to correlate the end-result with the initial clinical picture in the individual case has proved unrewarding, as in the great majority of previous studies of nephritis. The most that can be said is that of the cases ultimately found to have active or latent nephritis, a higher proportion had a marked hypertension in the initial stages than in the series as a whole. This is of no value in assessing the prognosis in the individual case, however; many cases with severe hypertension have recovered completely. It is probably true that the persistence of hypertension or gross oedema beyond the first few weeks are ominous signs and that cases which still show evidence of nephritis at the end of one year have mostly suffered permanent renal damage. Nevertheless, one case in this series recovered completely though her diastolic pressure never fell below $100 \mathrm{~mm}$. $\mathrm{Hg}$ in her first two months in hospital, while another recovered after four separate relapses with frank haematuria, spread over a period of 18 months. In the second case the evidence of recovery is based on three separate examinations at intervals over a period of one year, each examination including a blood urea and blood pressure estimation and an Addis count.

It remains true that to the anxious parents of a child in the early stages of nephritis we can talk only in terms of probabilities and can give no certain opinion on the outcome based on the findings in his particular case.

\section{Summary}

An attempt has been made to assess the long-term prognosis of nephritis in childhood by a study of 265 consecutive cases of nephritis admitted to the Royal Aberdeen Hospital for Sick Children over a period of 18 years.

Twenty cases $\left(7 \cdot 5^{\circ}\right)$ died in the initial illness, but it is shown that the mortality rate has fallen in recent years, largely owing to better control of infection. The mortality to be expected at present is in the neighbourhood of $3 \%$.

The fate of 178 survivors from the initial illness has been assessed from one to 13 years after the onset of the nephritis and $75 \%$ appear to have made a complete recovery.

Conflicting views on the prognosis of nephritis are discussed.

A re-examination of 34 cases with the use of Addis counts has shown no evidence of persistent renal damage in cases passed as healed on the strength of routine examination.

No correlation has been found between the initial clinical picture in the individual case and the ultimate result.

\section{A P P E N D I X}

\section{The Addis Count}

The sediment count first devised by Addis (1925) has been widely used in America in the follow-up of nephritis and many reports agree on its value in detecting minor degrees of renal damage. So far as I am aware, only Cass (1939) and Giles (1947) have reported the use of this technique in assessing the long-term prognosis of nephritis in childhood in this country.

As originally described by Addis, the count includes the estimation of the protein content and of the total number of red cells, white and epithelial cells and casts in a 12-hour specimen of urine collected after a period of fluid restriction to ensure the production of a concentrated and moderately acid specimen. He suggests that in the female catheterization is essential for accurate counts.

As repeated catheterization is obviously undesirable in children, and as accurate estimations of protein and white cells are of doubtful validity in non-catheter specimens in the female, I have performed only the red cell and cast counts with, in addition, a qualitative test for albumin. There is indeed no clear evidence that the white cell count and albumin estimation provide any additional information of value when the count is used as a measure of activity in nephritis. Lyttle (1933) and Snoke (1938) found that, with urine volumes up to 200 $\mathrm{ml}$. in the 12-hour period, a trace of albumin on qualitative test corresponded to a quantitative result within normal limits. Rubin, Rapoport and Waltz (1942) found that the red cell count showed the most constant and persistent abnormality and considered that, taken alone, it provided a satisfactory index of nephritic activity.

I have followed Addis's original technique in performing the counts with modification of the degree of fluid restriction as suggested for use with children by Giles (1947).

Counts performed on 20 normal children showed a range from 0 to 360,000 red cells and from 0 to 9,500 casts per 12-hour specimen, and I have accepted as the upper limits of normal the figures proposed by Lyttle 
(1933), Snoke (1938) and Giles (1947), namely, 600,000 red cells and 10,000 casts.

\section{REFERENCES}

Addis, T. (1925). J. Amer. med. Ass., 85, 163.

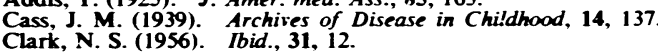

Craig, J., Clark, N. S. and Chalmers, J. D. (1949). Brit. med. J., $1,6$.

Ellis $A$ (1942) Lancet, 1, 1, 34 and 72.

Giles, M. D. (1947). Archives of Disease in Childhoud, 22, 232.

Hebert, H. J. (1952). J. Pediat., 40, 549.
Lawson, D. (1951). Brit. med. J., 1, 1423.

Murphy, F. D. and Rastetter, J. W. (1938). J. Amer. med. Ass.,

Payne, W. W6. and Illingworth, R. S. (1940). Quart. J. Med., 33 (n.s. 9), 37.

Rubin, M. I., Rapoport, M. and Waltz, A. D. (1942). J. Pediat., 20, 32.

Russell, C. V. (1949). Personal communication.

Snoke, A. W. (1937). Amer. J. Dis. Child., 53, 673.

(1938). J. Pediat., 12, 473.

(1939). Amer. J. Dis. Child., 57, 1373.

Tallerman, K. H. and Burkinshaw, J. H. (1939). Lancet, 1. 1255.

Thomson, T. R. (1949). Clin. Rep. Adelaidz Child. Ho.p., 1, 257. 